\title{
Pentoxifylline treatment and penile calcifications in men with Peyronie's disease
}

\author{
James F Smith $^{1,2}$, Alan W Shindel ${ }^{1}$, Yun-Ching Huang ${ }^{1}$, Raul I Clavijo ${ }^{1}$, Lawrence Flechner ${ }^{1}$, Benjamin N Breyer ${ }^{1}$, \\ Michael L Eisenberg ${ }^{1}$ and Tom F Lue ${ }^{1}$
}

This retrospective cohort study from a single clinical practice enrolled patients with evidence of calcified Peyronie's disease (PD) plaques detected on penile ultrasound at the time of initial presentation. The primary objective was to describe the effect of pentoxifylline (PTX) treatment on subtunical calcifications in men with PD. A PD-specific questionnaire was administered and sonographic evaluations were performed at baseline and follow-up visits. Descriptive statistics and $\chi^{2}$ analysis were used to characterize the effect of PTX on calcified tunical plaques. In all, 71 men (mean age: 51.9 years) with PD and sonographic evidence of calcification were identified. Of them, 62 of these men were treated with PTX for a mean duration of 1 year, and nine with vitamin E or no treatment. Improvement or stabilization in calcium burden at follow-up was noted in 57 (91.9\%) of men treated with PTX versus four (44.4\%) of those not treated with PTX $(P<0.001)$. PTX users were much less likely to have a subjective worsening of their clinical condition $(25.0 \%$ versus $78.3 \%, P=0.002)$. Treatment with PTX appeared to stabilize or reduce calcium content in PD plaques. A randomized controlled trial is warranted to further explore this effect.

Asian Journal of Andrology (2011) 13, 322-325; doi:10.1038/aja.2010.117; published online 22 November 2010

Keywords: penile calcifications; pentoxifylline; Peyronie's disease; treatment

\section{INTRODUCTION}

Peyronie's disease (PD) is a relatively common disorder in men (3-9\%) and a frequent (but not universal) cause of sexual distress. ${ }^{1,2}$ In its more severe and/or chronic forms, PD has been associated with calcium deposition in tunical plaques. The relative bother of calcium deposits in the penis varies, although calcified plaques tend to be more easily palpable and hence are presumably more likely to cause subjective distress. These plaques may also cause greater discomfort for men and their sexual partners.

A wide range of medical therapies has been used for $\mathrm{PD},{ }^{3-9}$ but there is no universally agreed upon standard medical treatment. Furthermore, medical treatment is generally thought to be efficacious only in the early phase of the disease, before plaque hardening and/or calcification has occurred. ${ }^{10}$ As such, the search for a pharmaceutical option that is effective, well tolerated, and effective in both the acute and chronic phase of PD continues.

Pentoxifylline (PTX) is a non-specific phosphodiesterase inhibitor with anti-inflammatory properties that has been used to treat claudication. ${ }^{11}$ PTX has also been used to decrease inflammation and fibrosis in kidney transplants, open heart surgery, dermatological conditions and after radiation injury. ${ }^{12-16}$ PTX has also been shown to reduce renal calcification in a rat mode of cisplatin-induced kidney injury. ${ }^{17}$ With respect to penile diseases, case reports have suggested that PTX may prevent intracavernosal fibrosis after priapism ${ }^{18}$ and decrease calcification in new-onset PD. ${ }^{19}$ In general, the side effects of PTX are mild and consist of nausea, dizziness and headache. ${ }^{11}$ These traits make PTX an interesting potential option for PD therapy.

Additional study is needed to determine the true utility of PTX as a treatment for PD. In the present retrospective cohort study, we investigate the utility of PTX as a treatment in men with PD and sonographic evidence of tunical calcification. We believe that this cohort represents a more severe and chronic subset of the PD population. Our primary outcome measure was quantitative and qualitative changes in the calcium content of the tunical plaque after PTX treatment versus no intervention or vitamin E monotherapy. Secondary outcome measures included patient assessment of treatment efficacy and clinical response.

\section{MATERIALS AND METHODS}

Study design and cohort description

This retrospective cohort study enrolled patients from a single clinical practice. At presentation, men diagnosed with PD completed a nonvalidated $\mathrm{PD}$-specific questionnaire detailing the past medical history, health-related behaviors and characteristics of their PD. Sonographic evaluation of the penis was performed on all men. Men with at least two penile ultrasounds and sonographic evidence of tunical calcifications at their first clinical visit were included. Men with congenital curvature were excluded. Institutional review board approval was obtained.

\footnotetext{
${ }^{1}$ Department of Urology, University of California, San Francisco, CA, USA and ${ }^{2}$ Department of Obstetrics, Gynecology, and Reproductive Sciences, University of California, San Francisco, CA 94143-0738, USA

Correspondence: Dr T F Lue (tlue@urology.ucsf.edu)

Received: 25 June 2010; Revised: 6 August 2010; Accepted: 26 August 2010; Published online: 22 November 2010
} 


\section{Main outcome measures}

Sonographic variables. Penile calcifications were identified using B-mode (gray scale) sonography on a General Electric Logiq P5 ultrasound machine with a $12-\mathrm{MHz}$ small-parts linear array probe. Measurements of calcification length and width were made in the longitudinal and transverse axes. The total area of calcification was determined by the product of the length and width of each calcified lesion. For men who had more than one calcification, the sum of each calcified area was used to determine a cumulative burden of calcification for each individual at baseline. The medical records of each subject were reviewed by an urologist to ascertain the presence of and the volume of tunical calcification for each patient. Uncertain findings in the medical record were adjudicated by two other authors. Post-treatment calcium burden was considered improved if there was a decrease in the number, size or density of sonographic calcifications. Calcium content was recorded as unchanged if there was no difference in size, number or density of calcification over time. A worsening in calcifications was defined as an increase in the size, number or density of calcifications.

\section{Predictor variable}

Treatments utilized. Treatment for PD was determined by prescription of any medication at the baseline or follow-up visit and recorded in the medical record. In general, PTX was prescribed to men at a dose of $400 \mathrm{mg}$ three times daily for an initial period of $4-6$ months. At the 4- to 6-month time point, men were reassessed with a clinical exam and gray scale or duplex ultrasound. Five men increased their dose to $800 \mathrm{mg}$ three times daily. Cumulative use of PTX was calculated by the sum of all the months using PTX from the first clinic visit to the last follow-up clinic visit.

$P D$ and sexual function characteristics. Duration of disease was determined by the difference between the date of presentation for evaluation and the date of onset of symptoms. Previous penile trauma or injury (yes/no), ability to maintain an erection, painful erections and degree of curvature (degrees) were determined by self-reported answers to the PD-specific questionnaire administered at the time of the initial evaluation for PD.

\section{Statistical analysis}

Descriptive statistics (for example, counts and means) and bivariate analyses were used to describe the study population and to assess the association between PTX use and calcification status. $\chi^{2}$ analysis was used to identify statistical differences between treatment groups. Because of small numbers in subgroups, a new variable was created by combining 'improvement' and 'no change' in calcifications over time to be compared with those patients who had worsening of their condition. All analyses were conducted using STATA 10 (Stata Corp., College Station, TX, USA). A significance level of $<0.05$ was considered significant and all tests were two-sided.

\section{RESULTS}

A total of 71 men with PD and sonographic evidence of penile calcification were identified. Of them, 62 had been treated with PTX (mean duration of treatment: 1.2 years) compared with nine who had not received PTX. Of the men who never took PTX, four used vitamin $\mathrm{E}$ and five used no medical treatment. The mean age of men in the cohort was 51.9 years with a mean duration of PD symptoms of 2.1 years (Table 1). The mean calcium burden at baseline for men in the cohort was $13.8 \mathrm{~mm}^{2}$ with an average of 1.6 discrete calcifications per individual. No statistically significant differences were observed between users and non-users of PTX.

Men taking PTX were more likely to have an improvement in their calcifications relative to men who did not take PTX (69.4\% versus $33.3 \%, P=0.03$; Table 2). Furthermore, men taking PTX were much more likely to have stabilization (that is, no change) or improvement in their calcium burden relative to men not taking PTX (91.9\% versus

Table 1 Baseline demographic and Peyronie's disease characteristics of men with calcifications at their initial evaluation and at least one follow-up ultrasound

\begin{tabular}{|c|c|c|c|c|c|c|c|c|}
\hline & & \multicolumn{2}{|c|}{ Overall } & \multicolumn{2}{|c|}{ No PTX } & \multicolumn{2}{|c|}{ PTX } & \multirow{2}{*}{ P value } \\
\hline & & $\mathrm{n}$ & $\%$ & $\mathrm{n}$ & $\%$ & $n$ & $\%$ & \\
\hline \multirow[t]{4}{*}{ Age (years) } & $<40$ & 10 & 14.1 & 1 & 11.1 & 9 & 14.5 & \\
\hline & $40-49$ & 14 & 19.7 & 3 & 33.3 & 11 & 17.7 & \\
\hline & $50-59$ & 36 & 50.7 & 5 & 55.6 & 31 & 50.0 & \\
\hline & $60-69$ & 11 & 15.5 & 0 & 0.0 & 11 & 17.7 & 0.44 \\
\hline \multirow[t]{2}{*}{ Able to maintain an erection } & No & 10 & 15.4 & 3 & 37.5 & 7 & 12.3 & \\
\hline & Yes & 55 & 84.6 & 5 & 62.5 & 50 & 87.7 & 0.06 \\
\hline \multirow[t]{2}{*}{ Painful erections } & No & 27 & 65.9 & 6 & 85.7 & 21 & 61.8 & \\
\hline & Yes & 14 & 34.2 & 1 & 14.3 & 13 & 38.2 & 0.22 \\
\hline \multirow[t]{2}{*}{ PTX treatment } & No & 9 & 12.7 & & & & & \\
\hline & Yes & 62 & 87.3 & & & & & \\
\hline \multicolumn{2}{|c|}{ Duration of PTX treatment (years. mean \pm s.d.) } & - & - & - & - & 1.2 & 1.1 & \\
\hline \multicolumn{2}{|c|}{ Duration of disease (years. mean \pm s.d.) } & 2.1 & 2.5 & 1.7 & 1.3 & 2.1 & 2.7 & 0.69 \\
\hline \multirow[t]{4}{*}{ Curvature $\left(^{\circ}\right)$} & $<30$ & 12 & 16.9 & 1 & 11.1 & 11 & 17.7 & \\
\hline & $30-59$ & 24 & 33.8 & 4 & 44.4 & 20 & 32.3 & \\
\hline & $\geqslant 60$ & 12 & 16.9 & 2 & 22.2 & 10 & 16.1 & \\
\hline & Unknown & 23 & 32.4 & 2 & 22.2 & 21 & 33.9 & 0.79 \\
\hline \multicolumn{2}{|l|}{ Penile deformity } & 30 & 42.9 & 5 & 55.6 & 25 & 41.0 & 0.41 \\
\hline \multicolumn{2}{|l|}{ Previous treatment for PD } & 63 & 88.7 & 9 & 100.0 & 54 & 87.1 & 0.25 \\
\hline \multicolumn{2}{|l|}{ History of penile injury } & 19 & 36.5 & 4 & 50.0 & 15 & 34.1 & 0.39 \\
\hline \multicolumn{2}{|c|}{ No. of calcifications (mean \pm s.d.) } & 1.6 & 1.2 & 1.9 & 1.3 & 1.6 & 1.2 & 0.49 \\
\hline \multicolumn{2}{|c|}{ Cumulative calcified area $\left(\mathrm{mm}^{2}\right.$, mean \pm s.d. $)$} & 13.8 & 23.1 & 12.4 & 8.6 & 14.0 & 25.1 & 0.86 \\
\hline
\end{tabular}

Abbreviation: PD, Peyronie's disease; PTX, pentoxifylline; —, No treatment for Peyronie's disease. 
Table 2 Change in calcifications in men taking PTX compared with men not taking PTX

\begin{tabular}{|c|c|c|c|c|c|c|}
\hline & \multicolumn{2}{|c|}{ Calcification improved } & \multicolumn{2}{|c|}{ Calcifications unchanged } & \multicolumn{2}{|c|}{ Calcifications unchanged or improvec } \\
\hline No PTX $(n=9)$ & $6(66.7)$ & $3(33.3)$ & $8(88.9)$ & $1(11.1)$ & $5(55.6)$ & $4(44.4)$ \\
\hline$P$ value & & 0.03 & & 0.43 & & $<0.001$ \\
\hline
\end{tabular}

Abbreviation: PTX, pentoxifylline.

Table 3 Change in subjective clinical condition in men taking PTX compared with men not taking PTX

\begin{tabular}{|c|c|c|c|c|c|c|}
\hline & \multicolumn{2}{|c|}{ Clinically improved } & \multicolumn{2}{|c|}{ Clinically unchanged } & \multicolumn{2}{|c|}{ Clinically unchanged or improved } \\
\hline No PTX $(n=8)$ & $6(75.0)$ & $2(25.0)$ & $8(100.0)$ & $0(0.0)$ & $6(75.0)$ & $2(25.0)$ \\
\hline$P$ value & & 0.04 & & 0.24 & & 0.002 \\
\hline
\end{tabular}

Abbreviation: PTX, pentoxifylline.

$44.4 \%, P<0.001)$. When men were queried about their subjective sense of clinical improvement, men taking PTX were more likely to experience a stabilization or improvement in their clinical condition compared with men not taking PTX (78.3\% versus $25.0 \%, P=0.002$; Table 3).

\section{DISCUSSION}

Men with calcified PD represent a difficult-to-treat population that has by and large been neglected in published reports of PD therapy. In this study, we report that PTX has efficacy in reducing penile calcium burden in men with PD and sonographic evidence of penile calcification. Furthermore, men taking PTX were less likely to experience worsening in their subjective assessment of their clinical condition.

A number of previous studies have reported declines in plaque size and consistency with medical therapies. Levine ${ }^{20}$ reported a substantial reduction in plaque size in 38 men treated with biweekly penile verapamil injections over a 6-month period; substantial plaque softening was also noted in treated men. Rehman et al. ${ }^{8}$ reported similar results in a single-blind randomized study of 14 men treated with intralesional verapamil. In a larger randomized clinical trial of 117 men, Hellstrom et al. ${ }^{4}$ reported greater rates of plaque shrinkage and softening in men treated with intralesional injection of interferon alpha-2b. However, a smaller trial of 30 men with PD were treated with intralesional interferon weekly for 12 weeks versus vitamin E 400 IU daily, as a control group did not demonstrate any significant differences between groups or changes over time. ${ }^{5}$ Most recently, Jordan ${ }^{21}$ reported on 25 patients with $\mathrm{PD}$ who were treated with intralesional injection of clostridial collagenase (two cycles of three injections). Significant declines in mean plaque length and width were noted at 3-, 6- and 9-month follow-up time points. Although these trials suggest a number of different treatments, which may be efficacious in managing penile plaques, calcification was not the primary end point in any of these and it is therefore unclear whether or not these modalities are useful in calcified PD. Indeed, for several of these trials, tunical calcification was not explicitly assessed or was an exclusion criterion for enrollment. $^{4,8,21}$

It has been demonstrated in vitro that cells derived from both normal tunica albuginea and PD plaque express osteogenic markers and have the capacity to undergo calcification. Incubation with tumor growth factor-beta, a known mediator of the PD phenotype, ${ }^{22}$ was shown to induce differentiation of cells into osteoblasts and myofibroblasts. This finding suggests that differentiation of cells along the osteoblast lineage is one possible mechanism for penile calcification in clinical PD. ${ }^{23}$

Although some data suggest that PTX blocks the tumor growth factor-beta pathways, ${ }^{24}$ the mechanism by which PTX might induce regression of calcified plaques is unclear. PTX has been shown to improve microcirculation through reducing blood viscosity. PTX increases erythrocyte deformability, decreases platelet aggregation and decreases fibrinogen levels. ${ }^{25,26}$ Furthermore, in an animal model, PTX was associated with increasing fibrinolytic activity in the peritoneum leading to a decrease in formation of peritoneal adhesions. ${ }^{27}$ This may be relevant to PD as it has been shown that fibrin can induce PD-like lesions in animal models. ${ }^{28}$ Therefore, along with its antifibrotic and anti-inflammatory properties, PTX may increase microcirculation in the site of fibrotic and calcified plaques, leading to faster plaque resolution and a decrease in calcification over time. Further study of mechanisms will be required to determine the actual activity of PTX in PD plaques.

Several limitations of this study are worth noting. Our relatively small sample size precluded a comprehensive multivariable analysis and the population was not randomized at baseline. Objective data on change in penile curvature, plaque volume, erectile function and erectile pain were not universally collected and therefore could not be included in the analysis. Because of this study design, it is possible that systematic differences between men taking PTX and those not taking PTX can explain some of the differences observed. It is also possible that men taking PTX were observed differentially relative to men not receiving PTX. Furthermore, unmeasured confounders could account for outcome differences between men who were or were not treated with PTX. As such, these results should be considered preliminary.

Despite these limitations, our study is unique in that it addresses the impact of PTX on calcium deposits in PD. To our knowledge, no previous study has documented a decline or stabilization in penile calcium burden with PTX therapy. This novel finding suggests that PTX may have particular efficacy in PD, even during the chronic phase of disease. This medication is cheap, well tolerated and has a strong basic science rationale to support its use. A randomized controlled trial evaluating the effect of PTX on calcifications and other clinical outcomes is needed to determine the role of PTX in the management of PD. 


\section{AUTHOR CONTRIBUTIONS}

JFS, AWS, BNB, MLE and TFL substantially contributed to conception and design. JFS, YCH, RIC, LF and TFL contributed to acquisition of data. JFS, AWS, and TFL analyzed the data. JFS, AWS and RIC drafted the manuscript. JFS, AWS, YCH, RIC, LF, BNB, MLE and TFL revised the article. JFS, AWS, YCH, RIC, LF, BNB, MLE and TFL contributed to the final approval of the article.

\section{COMPETING FINANCIAL INTERESTS}

The authors declare no conflict of interests.

\section{ACKNOWLEDGMENTS}

This work was supported in part by a research grant from the Sexual Medicine Society of North America.

1 Mulhall JP, Creech SD, Boorjian SA, Ghaly S, Kim ED et al. Subjective and objective analysis of the prevalence of Peyronie's disease in a population of men presenting for prostate cancer screening. J Urol 2004; 171: 2350-3.

2 Schwarzer U, Sommer F, Klotz T, Braun M, Reifenrath B et al. The prevalence of Peyronie's disease: results of a large survey. BJU Int 2001; 88: 727-30.

3 Safarinejad MR. Therapeutic effects of colchicine in the management of Peyronie's disease: a randomized double-blind, placebo-controlled study. Int J Impot Res 2004 16: $238-43$.

4 Hellstrom WJ, Kendirci M, Matern R, Cockerham Y, Myers L et al. Single-blind, multicenter, placebo controlled, parallel study to assess the safety and efficacy of intralesional interferon alpha-2B for minimally invasive treatment for Peyronie's disease. J Urol 2006; 176: 394-8.

5 Inal T, Tokatli Z, Akand M, Ozdiler E, Yaman O. Effect of intralesional interferon-alpha $2 \mathrm{~b}$ combined with oral vitamin E for treatment of early stage Peyronie's disease: a randomized and prospective study. Urology 2006; 67: 1038-42.

6 Cavallini G, Biagiotti G, Koverech A, Vitali G. Oral propionyl-L-carnitine and intraplaque verapamil in the therapy of advanced and resistant Peyronie's disease. BJU Int 2002; 89: 895-900.

7 Greenfield JM, Shah SJ, Levine LA. Verapamil versus saline in electromotive drug administration for Peyronie's disease: a double-blind, placebo controlled trial. J Urol 2007; 177: 972-5.

8 Rehman J, Benet A, Melman A. Use of intralesional verapamil to dissolve Peyronie's disease plaque: a long-term single-blind study. Urology 1998; 51: 620-6.

9 Safarinejad MR, Hosseini SY, Kolahi AA. Comparison of vitamin E and propionyl-Lcarnitine, separately or in combination, in patients with early chronic Peyronie's disease: a double-blind, placebo controlled, randomized study. J Urol 2007; 178 1398-403; discussion 403.

10 Pryor J, Akkus E, Alter G, Jordan G, Lebret T et al. Peyronie's disease. J Sex Med2004; 1: $110-5$.
11 Aviado DM, Porter JM. Pentoxifylline: a new drug for the treatment of intermittent claudication. Mechanism of action, pharmacokinetics, clinical efficacy and adverse effects. Pharmacotherapy 1984; 4: 297-307.

12 McCarty MF. Interleukin- 6 as a central mediator of cardiovascular risk associated with chronic inflammation, smoking diabetes, and visceral obesity: down-regulation with essential fatty acids, ethanol and pentoxifylline. Med Hypotheses 1999; 52: 465-77.

13 Noel C, Copin MC, Hazzan M, Labalette M, Susen S et al. Immunomodulatory effect of pentoxifylline during human allograft rejection: involvement of tumor necrosis factoralpha and adhesion molecules. Transplantation 2000; 69: 1102-7.

14 Tittelbach J, Graefe T, Wollina U. Painful ulcers in calciphylaxis-combined treatment with maggot therapy and oral pentoxyfillin. J Dermatolog Treat 2001; 12 211-4.

15 Boldt J, Brosch C, Lehmann A, Haisch G, Lang J et al. Prophylactic use of pentoxifylline on inflammation in elderly cardiac surgery patients. Ann Thorac Surg 2001; 71: 1524-9.

16 Delanian S, Porcher R, Balla-Mekias S, Lefaix JL. Randomized, placebo-controlled trial of combined pentoxifylline and tocopherol for regression of superficial radiationinduced fibrosis. J Clin Oncol 2003; 21: 2545-50.

17 Saad SY, Najjar TA, Alashari M. Role of non-selective adenosine receptor blockade and phosphodiesterase inhibition in cisplatin-induced nephrogonadal toxicity in rats. Clin Exp Pharmacol Physiol 2004; 31: 862-7.

18 Rajfer J, Gore JL, Kaufman J, Gonzalez-Cadavid N. Case report: avoidance of pal pable corporal fibrosis due to priapism with upregulators of nitric oxide. J Sex Med 2006; 3: 173-6.

19 Brant WO, Dean RC, Lue TF. Treatment of Peyronie's disease with oral pentoxifylline. Nat Clin Pract Urol 2006; 3: 111-5; quiz 6.

20 Levine LA. Treatment of Peyronie's disease with intralesional verapamil injection. J Urol 1997; 158: 1395-9.

21 Jordan GH. The use of intralesional clostridial collagenase injection therapy for Peyronie's disease: a prospective, single-center, non-placebo-controlled study. J Sex Med 2008; 5: 180-7.

22 El-Sakka Al, Hassoba HM, Pillarisetty RJ, Dahiya R, Lue TF. Peyronie's disease is associated with an increase in transforming growth factor-beta protein expression. J Urol 1997; 158: 1391-4.

23 Vernet D, Nolazco G, Cantini L, Magee TR, Qian A et al. Evidence that osteogenic progenitor cells in the human tunica albuginea may originate from stem cells: implications for Peyronie disease. Biol Reprod 2005; 73: 1199-210.

24 Ng YY, Chen YM, Tsai TJ, Lan XR, Yang WC et al. Pentoxifylline inhibits transforming growth factor-beta signaling and renal fibrosis in experimental crescentic glomerulonephritis in rats. Am J Nephrol 2009; 29: 43-53.

25 Bacher A, Eggensperger E, Koppensteiner R, Mayer N, Klimscha W. Pentoxifylline attenuates the increase in whole blood viscosity after transfusion. Acta Anaesthesio Scand 2005; 49: 41-6.

26 Strano A, Davi G, Avellone G, Novo S, Pinto A. Double-blind, crossover study of the clinical efficacy and the hemorheological effects of pentoxifylline in patients with occlusive arterial disease of the lower limbs. Angiology 1984; 35: 459-66.

27 Tarhan OR, Barut I, Sutcu R, Akdeniz Y, Akturk O. Pentoxifylline, a methyl xanthine derivative, reduces peritoneal adhesions and increases peritoneal fibrinolysis in rats. Tohoku J Exp Med 2006; 209: 249-55.

28 Davila HH, Ferrini MG, Rajfer J, Gonzalez-Cadavid NF. Fibrin as an inducer of fibrosis in the tunica albuginea of the rat: a new animal model of Peyronie's disease. BJU Int 2003; 91: 830-8. 